\title{
Meteor showers of comet C/1964 N1 (Ikeya)
}

\author{
L. Neslušan ${ }^{1}$ and M. Hajduková Jr. ${ }^{2}$ \\ ${ }^{1}$ Astronomical Institute, Slovak Academy of Science, 05960 Tatranská Lomnica, Slovakia \\ e-mail: ne@ta3.sk \\ ${ }^{2}$ Astronomical Institute, Slovak Academy of Science, Dúbravská cesta 9, 84504 Bratislava, Slovakia \\ e-mail: astromia@savba.sk
}

Received 14 February 2018 / Accepted 23 May 2018

\begin{abstract}
Aims. We intend to map the meteor complex of the long-period comet C/1964 N1 (Ikeya), which is a proposed parent body of the July $\xi$-Arietids, the meteor shower 533 in the IAU MDC list.

Methods. For five perihelion passages of the parent comet in the past, we modeled the associated theoretical stream, its parts, consisting of 10000 test particles each, and followed the dynamical evolution of these parts up to the present. We performed several simulations of the evolution, with various strengths of the Poynting-Robertson effect. At the end of each simulation, we analyzed the mean orbital characteristics of the particles that approached Earth orbit and thus created one or several showers. The showers were compared with their observed counterparts as separated from photographic and several video databases when the separation was successful.

Results. The modeled stream of C/1964 N1 typically approaches Earth orbit in four filaments that correspond to four showers. Their radiant areas are close to the apex of Earth's motion around the Sun. We confirm the generic relationship between the studied parent comet and the July $\xi$-Arietids. The comet also seems to be the parent of the $\epsilon$-Geminids, shower 23 , and we suspect a relationship between the comet and the $\xi$-Geminids, shower 718, although the relationship is rather uncertain. The real counterparts of three of the predicted showers were selected in the CAMS and SonotaCo databases. However, these real showers are diffuse, with relatively few members, and determination of their characteristics is therefore uncertain; the showers were separated into more than one single "modification". Confirmation of their existence will have to await considerably more numerous data.
\end{abstract}

Key words. comets: individual: C/1964 N1 - meteorites, meteors, meteoroids

\section{Introduction}

Periodic comets are expected to release small particles, meteoroids, from their nuclei. Since the escape velocity at release is negligible in comparison to the heliocentric velocity of the comet, the orbits of the meteoroids do not initially differ much from the orbit of the comet. The meteoroids thus orbit the Sun in a corridor they share with their parent. Nevertheless, the gravitational perturbations of planets and non-gravitational effects can later move these meteoroids to some alternative orbital corridors. The spatial structure of the meteoroid stream becomes filamentary, and the stream particles can collide with our planet in more than a single arc of its orbit.

The structure of the stream that occurs as a result of the dynamical evolution of the stream particles can be traced by modeling the stream and its orbital evolution. In this paper, we model the potential stream of long-period comet C/1964 N1 (Ikeya) in this way. The streams of long-period comets have not been modeled as often as streams associated with shortperiod parent bodies, such as comets 96P/Machholz (McIntosh 1990; Kaňuchová \& Neslušan 2007; Babadzhanov et al. 2008; Neslušan et al. 2013b; Abedin et al. 2018) or 12P/Pons-Brooks (Tomko 2015; Tomko \& Neslušan 2016), or asteroids such as 3200 Phaethon (Babadzhanov 1994; Beech 2002; Ryabova 2007, 2008; de León et al. 2010; Jakubík \& Neslušan 2015; Ryabova \& Rendtel 2018) or 196256 (2003 EH1; Babadzhanov et al. 2008, 2017; Neslušan et al. 2013a; Kasuga \& Jewitt 2015; Abedin et al. $2015)$. Comets with an orbital period, $P$, of several centuries are also known to produce meteoroid streams, for example, C/1861 G1 (Thatcher) with $P=415 \mathrm{yr}$. This is the parent body of the April Lyrids, shower 6 (Porubčan et al. 1992; Arter \& Williams 1997; Porubčan \& Kornoš 2008; Kornoš et al. 2015). C/1917 F1 (Mellish) with $P=145 \mathrm{yr}$ is the parent body of the December Monocerotids, shower 19 and, probably also of the April $\rho$-Cygnids, shower 348 (Ohtsuka 1989; Lindblad \& Olsson-Steel 1990; Hasegawa 1999; Vereš et al. 2011; Neslušan \& Hajduková 2014; Hajduková et al. 2015). C/1979 Y1 (Bradfield) with $P=$ $305 \mathrm{yr}$ is the parent body of the July Pegasids, shower 175 (Jenniskens 2006; Ueda 2012; Hajduková \& Neslušan 2017). We also refer to the showers by their number in the IAU MDC list ${ }^{1}$ (Jopek \& Kaňuchová 2014) to give a unique identification.

Comet C/1964 N1 has an orbital period equal to $391 \mathrm{yr}$ and moves in a low-inclined but retrograde orbit; therefore, the expected geocentric velocity of the associated meteors is very high. The meteoroids from the comet, if they are actually released, are expected to exhibit an intensive light effect when colliding with the Earth's atmosphere, which means that the meteors should be easily detected when they occur on the night sky.

According to recent work by Šegon et al. (2017), comet $\mathrm{C} / 1964 \mathrm{~N} 1$ is the parent body of the July $\xi$-Arietids, shower 533. The authors modeled the streams of several parent bodies using the method developed by Vaubaillon et al. (2005); see also Jenniskens \& Vaubaillon (2008).

\footnotetext{
https://www.ta3.sk/IAUC22DB/MDC2007/
} 
Consistent with several previous papers (e.g., Neslušan et al. 2013b), we use the words "complex" and "stream" as synonyms referring to the whole structure of meteoroids released from the parent body. The complex often consists of several "filaments". If the particles of a filament hit Earth, they can cause a meteor "shower" corresponding to the filament.

\section{Orbit of the parent comet}

In our study, we consider the orbit of comet C/1964 N1 with the orbital elements published in the JPL small-body browser (Giorgini et al. 1996) $^{2}$. This orbit, referred to as at epoch 1964 July $24.0 \quad($ JDT $=2438600.5)$, has these elements: $q=0.821752 \mathrm{au}, \quad e=0.984643, \quad a=53.5099303 \mathrm{au}, \quad \omega=$ $290.7618^{\circ}, \quad \Omega=269.9493^{\circ}, \quad i=171.9200^{\circ}, \quad$ and $T=$ 2438608.7111 (1964 August 1.2111). We refer to this orbit as the nominal orbit. The orbital period of the comet in the nominal orbit is $390.993 \mathrm{yr}$.

The uncertainity of the nominal orbit, determined in 1964, is not known. Therefore, it is not possible to trace the uncertainity of its past evolution. In our modeling of the theoretical stream, we did not consider any set of cloned orbits, only the nominal orbit itself. We integrated this orbit backward in time, down to $100000 \mathrm{yr}$. The past evolution of the orbital elements is shown in Figs. 1a-f.

The minimum distance of the pre-perihelion and postperihelion arcs of the C/1964 N1 orbit from Earth's orbit is shown in Fig. 1g. In some time intervals, the orbital arcs approached the orbit of our planet relatively closely; the mutual distance was shorter than 0.1 au or even shorter than $0.01 \mathrm{au}$. Currently, the arcs are relatively far from Earth's orbit; the pre-perihelion arc is at 0.19 au and the post-perihelion arc is at $0.41 \mathrm{au}$.

\section{Modeling the stream}

The theoretical stream of the studied comet, C/1964 N1, was modeled in the way suggested by Neslušan (1999), which was later slightly modified (Tomko \& Neslušan 2012). In the modeling, the orbit of the parent body is first integrated backward in time to the moment of perihelion passage, which occurred closest to an arbitrarily chosen time $t_{\mathrm{ev}}$. In the perihelion, a cloud of 10000 test particles around the parent body was modeled, and this entire assembly was integrated forward in time, up to the present. At the end of the integration, we selected the particles moving in the orbits that approach Earth's orbit within $0.05 \mathrm{au}$. They were used to predict the mean characteristics of the expected shower. A brief summary of the method can also be found in Neslušan et al. (2013b), Jakubík \& Neslušan (2015), and others. Since the method has been described several times, we do not repeat the description here.

After selecting the particles that approach Earth's orbit within $0.05 \mathrm{au}$, we searched for a corresponding shower in the meteor databases, specifically in (1) the International Astronomical Union (IAU) Meteor Data Center (MDC) photographic database, version 2013 (Porubčan et al. 2011; Neslušan et al. 2014), (2) the IAU MDC Cameras for Allsky Meteor Surveillance (CAMS) video database (Gural 2011; Jenniskens et al. 2011, 2016a,c,b; Jenniskens \& Nénon 2016), (3) the 2007-2015 SonotaCo video (SonotaCo 2009, 2016), and (4) the EDMOND video databases (Kornoš et al. 2014a,b). These databases contain 4873, 110521,208826 , and 145830 records on meteor orbits and geophysical data, respectively.

\footnotetext{
2 http://ssd.jpl.nasa.gov/sbdb.cgi
}

To identify a predicted shower with its potential counterpart in a given database, we attempted to separate the shower on the basis of the predicted mean orbit from the database using the so-called break-point method (Neslušan et al. 1995, 2013c). In this method, the dependence of the number of selected meteors of a shower on the limiting value of the Southworth-Hawkins (Southworth \& Hawkins 1963) D discriminant, $D_{\text {lim }}$, is analyzed. If a shower is present in the set, then the dependence $N=N\left(D_{\text {lim }}\right)$ has a convex behavior with a constant or almost constant part: a plateau. The break point is a critical point $N\left(D_{\text {lim }}\right)$ behavior, giving the most suitable limiting value for the D discriminant to select the densest part of a particular shower from the near orbital phase space. A similar method for isolating meteor showers from the sporadic background, emphasizing that a cutoff value chosen has to reflect the strength of the shower compared to the local sporadic background, has recently been suggested by Moorhead (2016).

Since the meteoroids move in the orbits with a low absolute inclination to the ecliptic, they hit Earth within a relatively long arc of its orbit, and the direction of the velocity vector of our planet changes during the period of shower activity. The geocentric radiant then changes correspondingly. To eliminate this effect, we illustrate the radiant positions in the ecliptic coordinate frame with the $x$-axis permanently directed to the Sun, that is, in the Sun-centered ecliptic coordinate frame. (If $\lambda$ is the ecliptic longitude of the radiant in the standard ecliptic coordinates and $\lambda^{\prime}$ is the ecliptic longitude of the radiant in the Sun-centered ecliptic coordinates, then $\lambda^{\prime}=\lambda-\lambda_{\odot}$, where $\lambda_{\odot}$ is the solar longitude of the meteor.)

To integrate the orbits of the theoretical particles, parent comet, and perturbing planets, we used integrator RA15 (Everhart 1985) within the software package MERCURY (Chambers 1999). The gravitational perturbations of eight planets, Mercury to Neptune, were taken into account.

In our simulations, the acceleration due to the Poynting-Robertson (P-R) effect was considered. The term "P-R effect" is here used to refer to the action of radial electromagnetic radiation pressure as well as to the velocity-dependent effects on the meteoroid particles. We used the improved formulas derived by Klačka (2014). A more detailed description is given in our previous paper (Hajduková \& Neslušan 2017), for example. Parameter $\beta$, being the ratio of the accelerations due to both the $\mathrm{P}-\mathrm{R}$ effect and the gravity of the Sun, was again regarded as a free parameter.

We created a series of models for various combinations of specific values of evolutionary time $t_{\mathrm{ev}}$ and parameter $\beta$. In reality, the stream consists of particles of various sizes and densities, and parameter $\beta$ therefore ranges in a wider interval of values. In addition, the particles are released at various times, therefore their evolutionary time is different and can also acquire a value from a wider interval. Hence, the particular model we created does not represent a whole stream. The model of a whole stream is a composition of partial models that give a good prediction of the corresponding real showers.

\section{Predicted showers}

Considering the evolutionary time, $t_{\mathrm{ev}}$, equal to $5,10,20,40$, and $80 \mathrm{kyr}$ and the $\mathrm{P}-\mathrm{R}$ effect parameter $\beta$ acquiring the values 0.00001, 0.0001, 0.001, 0.003, 0.005, 0.007, and 0.009, we constructed the theoretical streams of the studied comet for every possible combinations of $t_{\mathrm{ev}}$ and $\beta$ values and followed the dynamical evolution of the created stream until the present. 

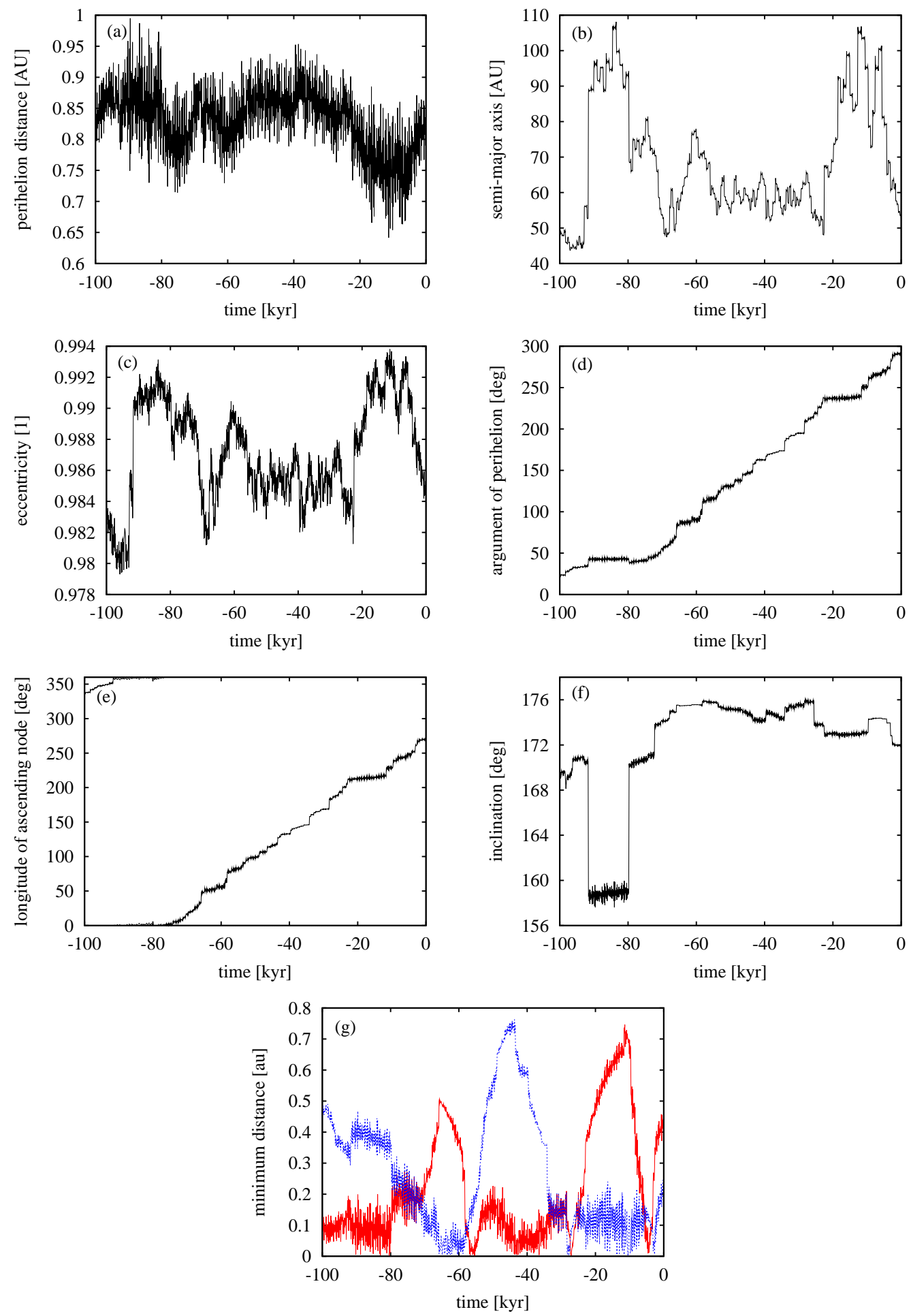

Fig. 1. Behavior of perihelion distance (panel a), semi-major axis (panel b), eccentricity (panel c), argument of perihelion (panel $d$ ), longitude of ascending node (panel e), and inclination to the ecliptic (panel f) of the nominal orbit of comet $\mathrm{C} / 1964 \mathrm{~N} 1$. The evolution of the minimum distance between the orbital arcs of comet C/1964 N1 and Earth is shown in plot (panel g). The minimum distance of the post-perihelion (pre-perihelion) arc is shown by the red solid (blue dashed) curve. All plots are constructed for the period from time 100000 yr before the present to the present.

For some values of evolutionary time, the P-R effect caused a deflection of the stream from the collisional course with Earth earlier or later than for the initially last considered value of $\beta=$ 0.009. To determine when the effect is strong enough to cause the complete deflection, we also created a few models with other values of $\beta$ than listed above $(\beta=0.006,0.008,0.010$, and 0.011$)$.
We also constructed some additional models when necessary (see their specification in Sect. 6.3).

The detailed distribution of the positions of geocentric radiants of the particles approaching Earth's orbit within $0.05 \mathrm{au}$ at the present is shown in Fig. 2 for four models in the Sun-centered ecliptic coordinates. The radiants are distributed 

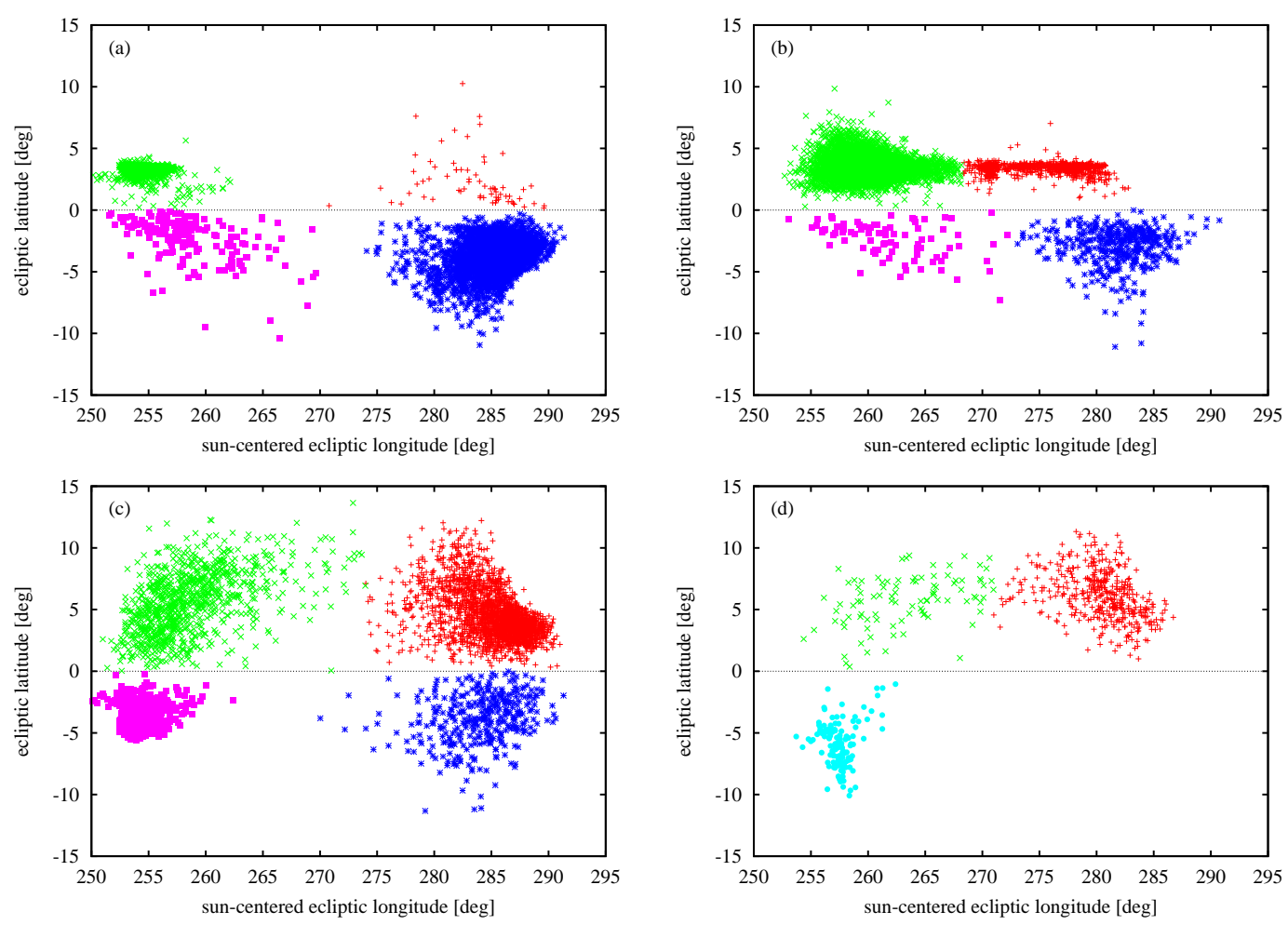

Fig. 2. Positions of geocentric radiants of theoretical particles grouped into five filaments of the meteoroid stream associated with comet $\mathrm{C} / 1964$ N1. The radiants of particles in the F1 (F2, F3, F4, and F5) filament are plotted with red pluses (green crosses, blue asterisks, violet full squares, and cyan full circles). The positions are shown for four models of the $\mathrm{C} / 1964 \mathrm{~N} 1 \mathrm{stream}$ characterized with $\left(t_{\mathrm{ev}}, \beta\right)$ equal to $(20,0.0001)($ panel $a)$, $(40,0.003)$ (panel b), $(80,0.00001)$ (panel c), and (80, 0.007) (panel $d$ ). To show the positions of the radiants, the Sun-centered ecliptical coordinate frame is used.

on both sides of the ecliptic. The northern and southern strands are further divided into two separate concentrations of the orbits in space, which is also seen in terms of radiants that are shown in the figure.

Taking into account this structure, we divided the stream mostly into four filaments, two (F1 and F2) with the radiants northward and two (F3 and F4) southward of the ecliptic. In a few models for $t_{\mathrm{ev}}=80 \mathrm{kyr}$ and high values of $\beta$ $(\beta=0.005,0.007,0.009$, and 0.010$)$, only a single southern filament occurred (Fig. 2d). In the Sun-centered ecliptic coordinates, the radiant area of this filament is similar to that of filament F4. A more detailed inspection reveals some systematic differences in the mean parameters (a higher absolute orbital inclination or lower solar longitude), however. Hence, we are not sure if this filament can be identified with $\mathrm{F} 4$, and we rather refer to it as F5. Its occurrence is discussed in Sect. 6.3.

In Figs. 3 and 4, the positions of the geocentric, Suncentered radiants of theoretical particles and the corresponding real showers are shown in some models. In more detail, the plots of Fig. 3 are related to filament F3 in the models with $\mathrm{P}-\mathrm{R}$ effect parameter $\beta=0.00001$ and a series of evolutionary times, $t_{\mathrm{ev}}$. The dispersion of the radiant area increases with increasing $t_{\mathrm{ev}}$. Owing to the larger dispersion, some particles are obviously deflected from the collisional course with Earth, therefore the number of particles in the filament decreases with the time.

The heliocentric radiants in Fig. 4 are related to filament $\mathrm{F} 2$ in the models for $t_{\mathrm{ev}}=40 \mathrm{kyr}$ and a series of the values of $\beta$-parameter. The $\mathrm{P}-\mathrm{R}$ effect on the particle dynamics in this filament is evident. The effect tends to move the articles away from the vicinity of Earth's orbit with increasing $\beta$. Only the particles in the core survive, therefore the extent of the radiant area is reduced.

We constructed plots like those in Figs. 3 and 4 for every filament in each model, where the identification of a predicted shower to a real shower separated at least from a single database was successful. Together with tabular data, these figures served to remove some identifications, which seemed to be successful on the basis of the $D$-criterion, but the predicted and observed radiant areas were unacceptably different (a more detailed description of the identification is given in Sect. 5).

According to Figs. 3 and 4, and many similar others that we do not show, there is mostly no perfect match between a given prediction and a real shower. However, the $\mathrm{P}-\mathrm{R}$ effect does not tend to improve the match significantly. The meteoroid particles in the C/1964 N1 stream are quite large and the effect is not very strong. In more detail, a good match can be found, typically, for $\beta$ up to the value of about 0.001 .

Furthermore, there is a vague symmetry of the filaments F1 and F4 and of F2 and F3 with respect to the apex of Earth's motion. The highest number of radiants of a given filament is found in a single quadrant (four filaments, F1-F4, in four quadrants) of the apex-centered ecliptical coordinate system. However, the distribution into specific quadrants is not exact. Some radiant points are beyond the quadrant pertaining to their filament and contaminate other filaments.

\section{Identification of predicted with real showers}

The mean orbits of the predicted meteor showers were used as the initial orbits in the iteration, made within the break-point method, to separate possible real counterparts of the showers 

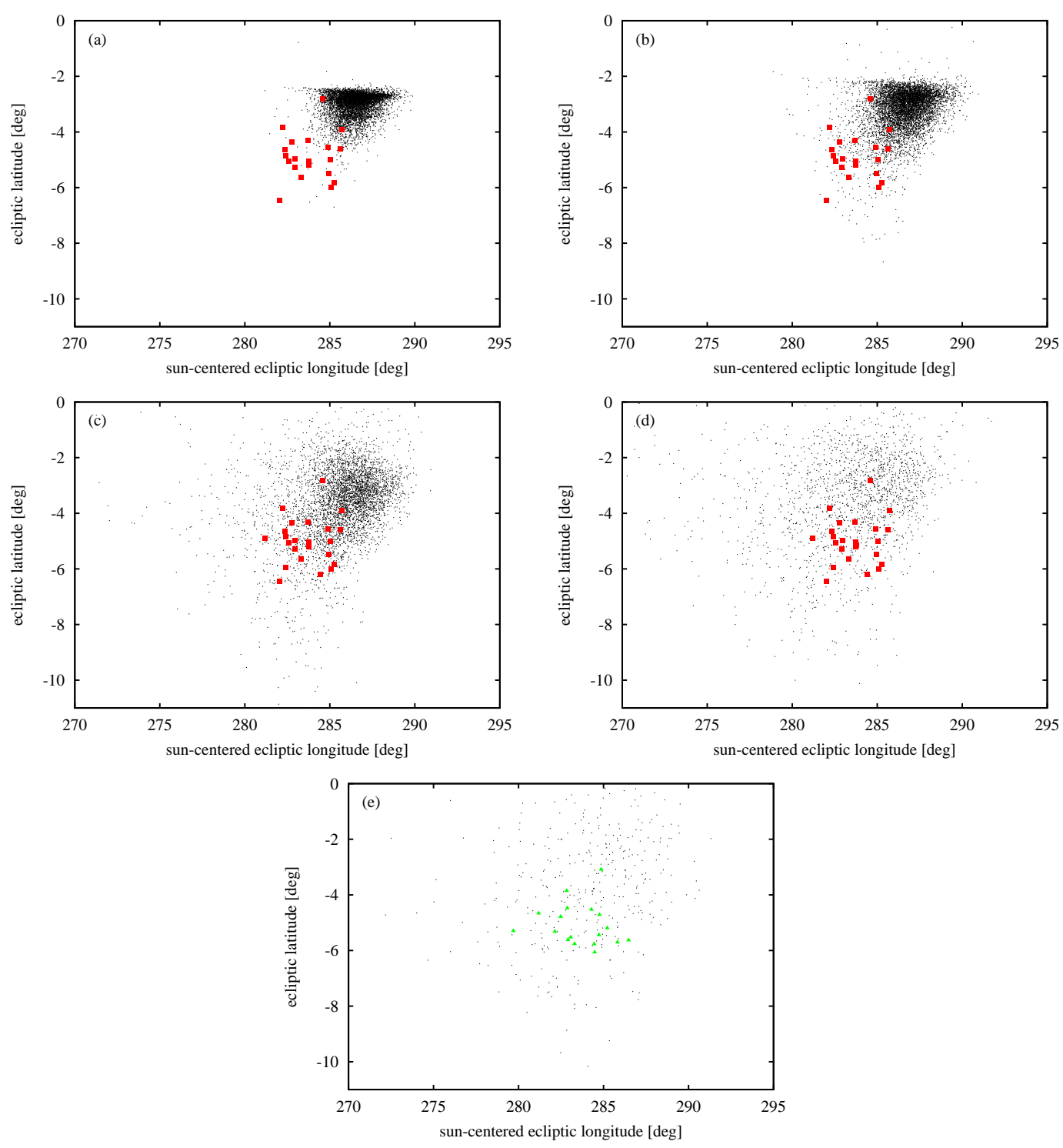

Fig. 3. Positions of geocentric radiants of theoretical particles (black dots) in the predicted filament F3 and corresponding real-shower meteors of the July $\xi$-Arietids,shower 533, separated from the CAMS-video (green triangles) and SonotaCo-video (red full squares) databases. The positions are shown in the models for the P-R effect parameter $\beta=0.00001$ and a series of evolutionary times $t_{\mathrm{ev}}=5$ (panel a), 10 (panel b), 20 (panel c), 40 (panel d), and $80 \mathrm{kyr}$ (panel e). To show the radiants, the Sun-centered ecliptic coordinate frame is used.

from the meteor catalogs. Unfortunately, the dependence $N=$ $N\left(D_{\text {lim }}\right)$ constructed within the method never exhibited a clear break point; therefore, it was hard to decide whether the separated set of meteors truly was a shower or only a random moderate accumulation of meteors in the given part of the orbital phase space. Hence, the reality of the mean orbits of the separated showers is rather uncertain.

The relationship between the predicted and separated showers is also mostly uncertain. The iteration often resulted in a real mean orbit that was very different from the predicted mean orbit. We calculated the Southworth-Hawkins D-discriminant between the predicted and corresponding real mean orbits and excluded all separated mean orbits with $D_{\mathrm{SH}}>0.2$. We also discarded some separated real showers if their geophysical data were too different from the predicted counterpart. In more detail, the difference in mean solar longitude should not exceed $\sim 20^{\circ}$, or the position of the predicted mean radiant should not be different from the real counterpart by more than a dozen degrees.
When comparing the predicted and real showers, we also considered our models with a relatively high value of the $\mathrm{P}-\mathrm{R}$ parameter $\beta$ and the video-meteor data. This may seem contradictory since the video techniques can detect relatively large particles and a high value of $\beta$ implies a small particle size. However, the relation between the size and parameter $\beta$ is still largely uncertain for the meteoroid material. Jakubík \& Neslušan (2015) separated the real Geminids, which have an ecliptic longitude of the radiant down to $254.5^{\circ}$. The radiants of these particles can reach this low value only for a $\beta$ parameter approaching 0.017. These Geminids were separated from the IAU MDC photographic as well as the video data, however. Hence, we can expect that some real particles with sizes corresponding to up to the highest considered $\beta$-value of 0.010 are present in the video data.

We also calculated the $D$-discriminant between every predicted and known mean orbit in the IAU MDC list of all showers and regarded as possibly related only orbits with $D_{\mathrm{SH}} \leq 0.20$. The criterion $D_{\mathrm{SH}} \leq 0.20$ is not very strict, therefore, it is 

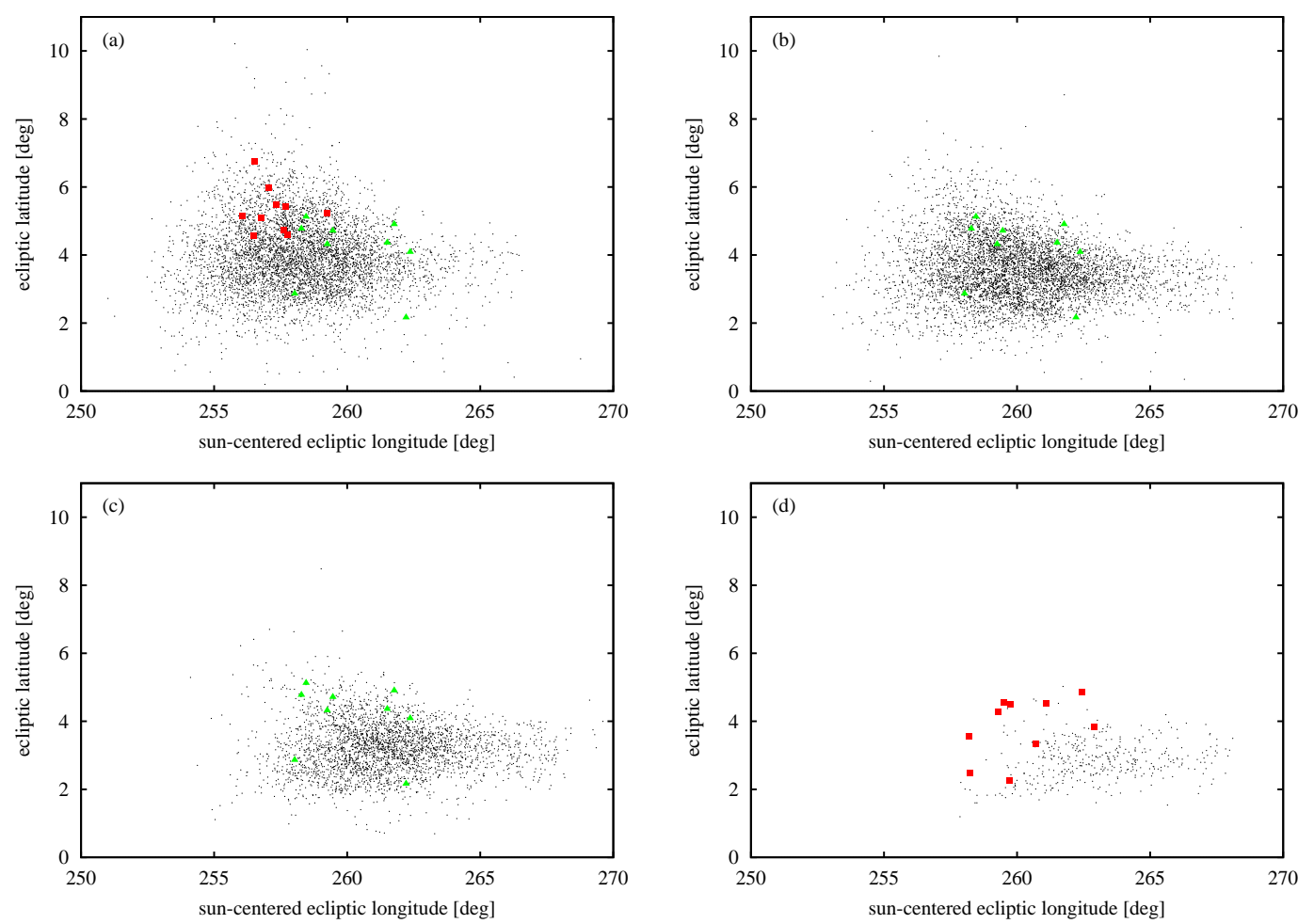

Fig. 4. Positions of geocentric radiants of theoretical particles (black dots) in the predicted filament F2 and corresponding real-shower meteors of the $\epsilon$-Geminids, shower 23, separated from the CAMS-video (green triangles) and SonotaCo-video (red full squares) databases. The positions are shown in the models for the evolutionary time $t_{\mathrm{ev}}=40 \mathrm{kyr}$ and a series of the values of $\mathrm{P}-\mathrm{R}$ effect parameter $\beta=0.001$ (panel a), 0.003 (panel b), 0.005 (panel c), and 0.007 (panel d). To show the radiants, the Sun-centered ecliptic coordinate frame is used.

not surprising that a single real shower, regardless of whether it was separated from a considered database or found in the IAU MDC list of showers, is identified with more than a single predicted filament. Fortunately, the search for the minimum value of $D_{\mathrm{SH}}$ between the given real shower and a predicted filament leads to a unique association of shower and filament.

Since a given filament consists of meteors on similar orbits, the orbital similarity and its evaluation with the help of $D$-discriminant is a reasonable way to identify the predicted filaments with the observed showers. In this context, we point out the circumstance that all predicted filaments of the C/1964 N1 stream have a low absolute inclination to the ecliptic $\left(|i| \lesssim 10^{\circ}\right)$, and consequently, the long orbital arcs of stream meteoroids are situated in the vicinity of Earth's orbit. Thus, the large difference in mean argument of perihelion and mean longitude of ascending node can naturally occur. Moreover, the meteoroids of a given filament can collide with our planet during a relatively long period. Our tolerance of $\sim 20^{\circ}$ in solar longitude, mentioned above, is therefore likely acceptable.

The differences of several degrees in mean solar longitude, mean right ascension, and mean declination of the radiant or those of several tenths of an astronomical unit in perihelion distance occur not only between the predicted and corresponding real showers, but also between the corresponding real showers, as separated by us in this work based on various catalogs, and between the real showers as separated from a catalog and the corresponding showers in the IAU MDC list of showers, which are also mutually different when determined by various authors. All these differences indicate that no stricter criterion of a shower identity than we considered can be used for the showers originating in C/1964 N1.

\section{Specific filaments}

\subsection{Filaments $F 3$ and F1, July $\xi$-Arietids}

We can clearly identify theoretical filament F3 with shower July $\xi$-Arietids, shower 533 in the IAU MDC list. This identification is consistent with the result obtained by Šegon et al. (2017). In our current study, the similarity between the mean orbit of the July $\xi$-Arietids and the predicted mean orbit of F3 is characterized by the very low $D$-discriminant $D_{\mathrm{SH}}=0.053$ when we consider the mean orbit determined by Šegon et al. (2014) and $D_{\mathrm{SH}}=0.070$ for the mean orbit determined by Jenniskens et al. (2016c). A slightly higher value, $D_{\mathrm{SH}}=0.078$, characterizes the similarity when we compare our filament F3 and the mean orbit derived by Kornoš et al. (2014b).

A real shower corresponding to filament F3, its models especially for $t_{\mathrm{ev}}=20 \mathrm{kyr}$, was also found in the SonotaCo video data and IAU MDC CAMS data. No shower corresponding to F3 was found in the IAU MDC photographic and EDMOND video data sets. The agreement between the predicted and real showers is better for $t_{\mathrm{ev}} \geq 20 \mathrm{kyr}$ than for a shorter evolutionary time. Hence, we can conclude that the age of the July $\xi$-Arietids is at least $20000 \mathrm{yr}$. Inspection of models for $t_{\mathrm{ev}}=80 \mathrm{kyr}$, however, implies that the age is younger than this. Comparing the predicted filament F3 to the real showers also implies that the real meteoroids were not much influenced by the P-R effect. Their size corresponds to $\beta \geqq 0.001$.

The radiant area of filament F3 is situated below the projection of the ecliptic on the celestial sphere. Filament F1 has a very similar mean orbit, but its radiant area is situated northward of the ecliptic. Hence, F1 can be regarded as a northern branch of the southern F3. Some showers with mean characteristics 
resembling those of F1 were found in the IAU MDC CAMS video as well as in the SonotaCo video databases. However, these real showers were predicted only with the help of models with $t_{\mathrm{ev}}=80$ and are very dispersed. They are also uncertain because the threshold used for their separation was found to be high, from 0.32 to 0.40 . Because of this uncertainty, we do not yet name these newly found showers. No shower in the IAU MDC list was identified with F1.

\subsection{Filaments F2 and F4, $\epsilon$-Geminids}

Filaments F1 and F3 can be regarded as the northern and southern branches of the same stream. Similarly, filaments F2 and F4 are such branches, F2 is the northern and F4 the southern branch. While filament F4 is not the most similar filament to any shower in the IAU MDC list of all showers, filament F2 can probably be identified with the $\epsilon$-Geminids, shower 23 , especially with the mean orbit of this shower as determined by Cook (1973).

Filament F2 probably has its counterpart in the IAU MDC CAMS data. Furthermore, we found a certain similarity of the mean orbit of F2 and three modifications of a corresponding real shower in the SonotaCo data. We must be careful with this identification, however, since the predicted solar longitude is up to $20^{\circ}$ higher. The matching degree of the predicted and real heliocentric radiant positions in F2 is shown in Fig. 4, where these positions are plotted in the models for $t_{\mathrm{ev}}=40 \mathrm{kyr}$ and a series of the $\mathrm{P}-\mathrm{R}$ effect parameter $\beta$. The predicted shower matches its real counterpart for a wide interval of $\beta$-values, from 0 to about 0.005 . No real counterpart of filament F4 was found in the considered databases or in the IAU MDC list.

\subsection{Filament F5}

In the theoretical models for $t_{\mathrm{ev}}=80 \mathrm{kyr}$ and $\beta \geq 0.005$, filaments F3 and F4, with radiant areas below the ecliptic, disappeared. Instead of these filaments, a single filament, F5, appeared with an area of the standard geocentric radiant in the region near the border of F3 and F4. If the radiants are plotted in the Suncentered ecliptic coordinates, the radiant area is almost the same as the radiant area of F4 (see Fig. 2d). Nevertheless, the differences in some other parameters do not disappear, therefore it is reasonable to classify F5 as an extra filament.

The occurrence of this irregular filament is most likely a consequence of the considerable change in inclination of the parent-comet orbit, which can be observed in Fig. 1f. In the period from about -92 to $-80 \mathrm{kyr}$, the inclination decreased from $\sim 170^{\circ}$ to $\sim 158^{\circ}$ (i.e., the absolute inclination increased from $\sim 10^{\circ}$ to $\sim 22^{\circ}$; the orbit was at a larger distance from the ecliptic). This is the effect of the temporary capture of C/1964 N1 into the Kozai resonance during that period.

The orbital evolution of stream meteoroids was likely similar and, hence, the dynamical behavior of the stream in a more distant past can be expected to be different from its behavior in a later period. To support this vague deduction based on the evolution of the parent-body orbit, we also created several additional models. Since filament F5 in the models for $t_{\mathrm{ev}}=80 \mathrm{kyr}$ is most abundant in the model for $\beta=0.007$, we constructed a set of models for $\beta=0.007$ and a sequence of values of $t_{\mathrm{ev}}$ equal to 50, $60,70,75,78,82,85$, and $90 \mathrm{kyr}$.

No single meteor was predicted in any orbit within $0.05 \mathrm{au}$ of Earth's orbit at the present time in the models for $t_{\mathrm{ev}}=90,85$, or $82 \mathrm{kyr}$, but when we considered the particles released from the parent $80 \mathrm{kyr}$ ago, some of them dynamically evolved to the present-day filament F5. The particles released $78 \mathrm{kyr}$ ago form a filament that can be identified as F5 and is predicted to be the dominant structure (except for F5, only a few meteors of F1 approached the orbit of our planet) at the present time. The stream that was later released from the parent, $75 \mathrm{kyr}$ and $60 \mathrm{kyr}$ ago, did not intersect Earth's orbit. The model for $t_{\mathrm{ev}}=60 \mathrm{kyr}$ predicts filament F1, but with only a few members. This absence of the stream at Earth's orbit was interrupted for a period: filaments F1, F2, and F5 (and a weak F3) occur again in the model at $t_{\mathrm{ev}}=70 \mathrm{kyr}$.

The filamentary structure becomes "normal", consisting of the above described filaments F1 to F4, when the meteoroids are released in time $\sim 50 \mathrm{kyr}$ before the present and later. In the model for $t_{\mathrm{ev}}=50 \mathrm{kyr}$, the filamentary structure is similar to that for $t_{\mathrm{ev}}=40 \mathrm{kyr}$ (and $\beta=0.007$ ), except for the circumstance that $\mathrm{F} 1$ is more abundant than $\mathrm{F} 2$ for $t_{\mathrm{ev}}=50 \mathrm{kyr}$.

No real counterpart of F5 was separated in the considered databases. We found only a vague similarity of its mean characteristics with the mean characteristics of $\xi$-Geminids, shower 718 , which was found by Jenniskens et al. (2016a). The match is poor in mean solar longitude (difference between the predicted and observed mean values $\left.\sim 15^{\circ}\right)$, mean right ascension $\left(\sim 10^{\circ}\right)$, mean declination $\left(\sim 5^{\circ}\right)$, and mean inclination $\left(\sim 10^{\circ}\right)$. Moreover, while Jenniskens et al. gave the mean semi-major axis as 5.8 au, we predict 337.1 au. This quantity can differ widely even for a well-established relationship, however.

\section{Conclusions}

We modeled a theoretical stream of comet C/1964 N1 (Ikeya). The models were characterized with a variety of free parameters, evolutionary time, and strength of the Poynting-Robertson drag $\beta$ to predict a part or parts of the stream that can collide with Earth. This means that we aimed to predict a meteor shower (or several of them) of the comet.

Our simulations imply the existence of four distinct filaments of the C/1964 N1 stream that might be observed in Earth's atmosphere as four individual showers. We labeled them F1 to F4. We found an indication that the orbit of the parent comet and its stream rapidly evolved in a period that ended about $50 \mathrm{kyr}$ ago. During this period, the orbital corridors of individual filaments occupied a different space.

Since our modeling ended with a positive result (many particles occurred in the vicinity of Earth's orbits at the present time), we then separated some corresponding showers from the considered databases of real meteors and compared the prediction with the observation. We also searched for the corresponding showers in the IAU MDC shower list.

We note, however, that the separation of real showers corresponding to the predicted ones was often not clear. When we started the iteration procedure from different initial orbits, we obtained several modifications of, most probably, the same real shower. The separated real showers contain few members, 28 at most, many of them consist of 10 or fewer members.

The diffuse real counterpart of filament F3 was found in the video data, especially in the SonotaCo database. In the IAU MDC list, this filament is clearly identified with the July $\xi$-Arietids, shower 533. This identification has previously also been made by Šegon et al. (2014, 2017). The identification of F2 with $\epsilon$-Geminids, shower 23 that we made is less certain. Its real counterpart was identified mainly in the CAMS data. A real counterpart of filament F1 was also found in the CAMS and SonotaCo data. We suspect, moreover, that filament F5 might be a prediction of the $\xi$-Geminids, shower 718 . This shower 
also has meteoroids on retrograde orbits and a similar mean argument of perihelion and mean longitude of the ascending node. The correspondence of some parameters is questionable, however, although it is not clearly excluded. No real counterpart of filament F4 was found in the databases or in the IAU MDC list.

No real shower corresponding to F4 was observed, probably because only relatively few particles are predicted to approach Earth's orbit and thus collide with our planet. This number is an order of magnitude lower than the numbers in filaments F3 (in the models for $t_{\mathrm{ev}}<80 \mathrm{kyr}$ ), F2 (for $t_{\mathrm{ev}}>5 \mathrm{kyr}$ and a relatively low numbers are predicted for $t_{\mathrm{ev}}=20$ and $80 \mathrm{kyr}$ ), and F1 (for $t_{\mathrm{ev}}>20 \mathrm{kyr}$ ). We can expect that the abundance of a real shower is proportional to the prediction. Consequently, if the databases contain only a few tens of meteors of the real counterparts of F1 to F3, these data can be expected to contain only a few meteors of F4 and F5 with the predicted number of the same magnitude as F4, and so showers with only a few particles cannot be separated from the databases.

Analyzing the agreement between the predicted and real showers, we can conclude that the meteoroids in the C/1964 N1 stream are older than about $20000 \mathrm{yr}$. The particles in filaments F1 to F4, which can collide with Earth at the present, could be released from the parent-body surface continuously in the period ranging from 50000 to $20000 \mathrm{yr}$ in the past. Some older particles, with ages of up to $80000 \mathrm{yr}$, might also survive in the stream; they were ejected from the parent's surface during some specific short time intervals, however. The particles in filament F5 could be released only during these intervals.

Filaments $\mathrm{F} 1$ to $\mathrm{F} 4$ should mostly contain relatively large particles corresponding to a $\mathrm{P}-\mathrm{R}$ parameter $\beta \lesssim 0.001$. Only filament F5, if its real counterpart exists, contains Earth-hitting particles that are small, corresponding to $0.005 \lesssim \beta \lesssim 0.010$.

The radiant areas of four showers corresponding to $\mathrm{F} 1-\mathrm{F} 4$ of the stream of C/1964 N1 are close to the apex of the motion of Earth around the Sun. Among the showers studied by us or other authors, this property is exceptional. No other stream has been found with such a configuration of radiant areas of its showers.

In conclusion, comet C/1964 N1 is an active parent body of at least one and possibly more real showers. The study of its stream is worth to be repeated when more extensive meteor data are collected.

Acknowledgements. This article was supported by the realization of Project ITMS No. 26220120029, based on the supporting operational research and development program financed from the European Regional Development Fund. The work was also supported, in part, by the VEGA - the Slovak Grant Agency for Science, grants No. 2/0037/18, and by the Slovak Research and Development Agency under the contract No. APVV-16-0148.

\section{References}

Abedin, A., Spurný, P., Wiegert, P., et al. 2015, Icarus, 261, 100

Abedin, A., Wiegert, P., Janches, D., et al. 2018, Icarus, 300, 360

Arter, T. R., \& Williams, I. P. 1997, MNRAS, 286, 163

Babadzhanov, P. B. 1994, in 75 Years of Hirayama Asteroid Families: The Role of Collisions in the Solar System, eds. Y. Kozai, R. P. Binzel, \& T. Hirayama, ASP Conf. Ser., 63, 168

Babadzhanov, P. B., Williams, I. P., \& Kokhirova, G. I. 2008, MNRAS, 386 2271
Babadzhanov, P. B., Kokhirova, G. I., Williams, I. P., \& Obrubov, Y. V. 2017, A\&A, 598, A94

Beech, M. 2002, MNRAS, 336, 559

Chambers, J. E. 1999, MNRAS, 304, 793

Cook, A. F. 1973, NASA Special Publication, 319, 183

de León, J., Campins, H., Tsiganis, K., Morbidelli, A., \& Licandro, J. 2010, A\&A, 513, A26

Everhart, E. 1985, in Dynamics of Comets: Their Origin and Evolution: Proceedings of IAU Colloq, 11 1984, eds. A. Carusi, \& G. B. Valsecchi (Dordrecht: Reidel), Astrophysics and Space Science Library

Giorgini, J. D., Yeomans, D. K., Chamberlin, A. B., et al. 1996, BASS, 28, 1158

Gural, P. S. 2011, in Proceedings of the 29th International Meteor Conference (Armagh, Northern Ireland), 2010, 28

Hajduková, M., \& Neslušan L. 2017, A\&A, 605, A36

Hajdukova, M., Rudawska, R., Kornos, L., \& Toth, J. 2015, in Planetary and Space Science, 118, 28

Hasegawa, I. 1999, in Meteroids 1998, eds. W. J. Baggaley \& V. Porubčan (Bratislava, Slovakia: Astronomical Institute of the Slovak Academy of Sciences) 177

Jakubík, M., \& Neslušan, L. 2015, MNRAS, 453, 1186

Jenniskens, P. 2006, Meteor Showers and Their Parent Comets (Cambridge, UK: Cambridge University Press)

Jenniskens, P., \& Nénon, Q. 2016, Icarus, 266, 371

Jenniskens, P., \& Vaubaillon, J. 2008, AJ, 136, 725

Jenniskens, P., Gural, P. S., Dynneson, L., et al. 2011, Icarus, 216, 40

Jenniskens, P., Nénon, Q., Albers, J., et al. 2016a, Icarus, 266, 331

Jenniskens, P., Nénon, Q., Gural, P. S., et al. 2016b, Icarus, 266, 355

Jenniskens, P., Nénon, Q., Gural, P. S., et al. 2016c, Icarus, 266, 384

Jopek, T. J., \& Kaňuchová, Z. 2014, Meteoroids 2013, 353

Kaňuchová, Z., \& Neslušan, L. 2007, A\&A, 470, 1123

Kasuga, T., \& Jewitt, D. 2015, AJ, 150, 152

Klačka, J. 2014, MNRAS, 443, 213

Kornoš, L., Koukal, J., Piffl, R., \& Tóth, J. 2014a, in Proceedings of the International Meteor Conference, Poznan, Poland, 22-25 August 2013, eds. M. Gyssens, P. Roggemans, \& P. Zoladek, 23

Kornoš, L., Matlovič, P., Rudawska, R., et al. 2014b, Meteoroids 2013, 225

Kornoš, L., Tóth, J., Porubčan, V., et al. 2015, Planet. Space Sci., 118, 48

Lindblad, B. A., \& Olsson-Steel, D. 1990, Bull. Astr. Inst. Czechosl., 41, 193

McIntosh, B. A. 1990, Icarus, 86, 299

Moorhead, A. V. 2016, MNRAS, 455, 4329

Neslušan, L. 1999, A\&A, 351, 752

Neslušan, L., \& Hajduková, M. 2014, A\&A, 566, A33

Neslušan, L., Svoreň, J., \& Porubčan, V. 1995, Earth Moon Planets, 68, 427

Neslušan, L., Hajduková, M., \& Jakubík, M. 2013a, A\&A, 560, A47

Neslušan, L., Kaňuchová, Z., \& Tomko, D. 2013b, A\&A, 551, A87

Neslušan, L., Svoreň, J., \& Porubčan, V. 2013c, Earth Moon Planets, 110, 41

Neslušan, L., Porubčan, V., \& Svoreň, J. 2014, Earth Moon Planets, 111, 105

Ohtsuka, K. 1989, WGN, J. Int. Meteor Organ., 17, 93

Porubčan, V., \& Kornoš, L. 2008, Earth Moon Planets, 102, 91

Porubčan, V., Štohl, J., \& Svoreň, J. 1992, Contrib. Astron. Obs. Skalnate Pleso, 22, 25

Porubčan, V., Svoreň, J., Neslušan, L., \& Schunová, E. 2011, in Meteoroids: The Smallest Solar System Bodies, eds. W. J. Cooke, D. E. Moser, B. F. Hardin, \& D. Janches (Washington D.C.: NASA) 338

Ryabova, G. O. 2007, MNRAS, 375, 1371

Ryabova, G. O. 2008, in European Planetary Science Congress 2008, 226

Ryabova, G. O., \& Rendtel, J. 2018, MNRAS, 475, L77

Šegon, D., Gural, P., Andreić, Ž., et al. 2014, WGN, J. Int. Meteor Organ., 42, 57

Šegon, D., Vaubaillon, J., Gural, P. S., et al. 2017, A\&A, 598, A15

SonotaCo. 2009, WGN, J. Int. Meteor Organ., 37, 55

SonotaCo. 2016, WGN, J. Int. Meteor Organ., 44, 42

Southworth, R. B., \& Hawkins, G. S. 1963, Smithsonian Contributions to Astrophysics (Washington D.C.: Smithsonian Institution), Vol. 7, 261

Tomko, D. 2015, Planet. Space Sci., 118, 35

Tomko, D., \& Neslušan, L. 2012, Earth Moon Planets, 108, 123

Tomko, D., \& Neslušan, L. 2016, A\&A, 592, A107

Ueda, M. 2012, WGN, J. Int. Meteor Organ., 40, 59

Vaubaillon, J., Colas, F., \& Jorda, L. 2005, A\&A, 439, 761

Vereš, P., Kornoš, L., \& Tóth, J. 2011, MNRAS, 412, 511 